\title{
Hemograma e perfil bioquímico sérico, inclusive hemogasométrico, de bezerros infectados experimentalmente com Salmonella Dublin
}

[Blood cell counts and serum biochemical profile, including blood gas levels, in Salmonella Dublin-infected calves]

\author{
D.G. Silva ${ }^{1}$, P.R.L. Silva ${ }^{2}$, J.J. Fagliari ${ }^{3 *}$ \\ ${ }^{1}$ Aluna de pós-doutorado - FCAV-UNESP - Jaboticabal, SP \\ ${ }^{2}$ Médico veterinário autônomo - FCAV-UNESP - Jaboticabal, SP \\ ${ }^{3}$ Faculdade de Ciências Agrárias e Veterinárias - UNESP \\ Via de Acesso Prof. Paulo Donato Castellane, s/n \\ 14884-900 - Jaboticabal, SP
}

\begin{abstract}
RESUMO
O objetivo do estudo foi avaliar o hemograma e o perfil bioquímico sérico, inclusive hemogasométrico, de bezerros infectados experimentalmente com Salmonella Dublin. Foram utilizados 12 bezerros sadios da raça Holandesa com 10 a 15 dias de idade, distribuídos aleatoriamente em dois grupos experimentais: grupocontrole $(n=6)$ e grupo infectado com $10^{8}$ UFC de Salmonella Dublin $(n=6)$. Os bezerros foram submetidos ao exame físico diário, e as amostras de sangue foram coletadas minutos antes da inoculação (0h) e 24, 48, 72, 96, 120, 144 e 168h após a inoculação. Além do hemograma e das análises hemogasométricas, foram mensuradas as atividades séricas das enzimas aspartato aminotransferase (AST), fosfatase alcalina (ALP), creatina cinase (CK), gamaglutamiltransferase (GGT) e lactato desidrogenase (LDH), e os teores de albumina, bilirrubinas, cálcio total, cálcio ionizado, sódio, potássio, cloretos, creatinina, ferro, fibrinogênio, fósforo, glicose, magnésio, proteína totais e ureia. As principais alterações foram: redução das concentrações de albumina, ferro, glicose, magnésio e proteína total, aumento do teor de fibrinogênio, leucocitose e acidose metabólica e hiponatremia.
\end{abstract}

Palavras-chave: bezerro, Salmonella Dublin, diarreia, hemograma, eletrólitos

\begin{abstract}
The blood cell counts and biochemical profile, including blood gas levels, were evaluated, in Salmonella Dublin-infected calves. Twelve healthy 10 to 15 -day old Holstein calves were randomly allotted into two groups: control $(n=6)$ and group orally infected with $10^{8} \mathrm{CFU}$ Salmonella Dublin $(n=6)$. The calves were submitted to physical examination and the blood samples were taken just before the inoculation (Oh) and at 24, 48, 72, 96, 120, 144, and 168h later. Besides, blood cell counts, blood gas levels, and the serum concentrations of aspartate aminotransferase (AST), alkaline phosphatase (ALP), creatine kinase (CK), gammaglutamyltransferase (GGT), lactate desidrogenase $(L D H)$, albumin, bilirubin, total calcium, ionic calcium, sodium, potassium, chlorides, creatinine, iron, fibrinogen, phosphorus, glucose, magnesium, total protein, and urea were measured. The main findings included decrease in albumin, iron, glucose, magnesium, and total protein concentrations, and increase in fibrinogen level, leukocytosis, metabolic acidosis, and hyponatremia.
\end{abstract}

Keywords: calves, Salmonella Dublin, diarrhea, hemogram, electrolytes

\section{INTRODUÇÃO}

A diarreia neonatal é considerada um dos principais problemas sanitários em rebanhos bovinos, causando grandes prejuízos econômicos

Recebido em 16 de novembro de 2009

Aceito em 31 de março de 2010

*Autor para correspondência (corresponding author)

E-mail: fagliari@fcav.unesp.br não somente pela alta taxa de mortalidade, mas também pelos custos com tratamento e prejuízo ao crescimento (Constable et al., 1996; Barrington et al., 2002). Embora grande número de agentes etiológicos possa estar envolvido com as enterites neonatais, as infecções por 
Salmonella, principalmente pelos sorotipos Dublin e Typhimurium, são as principais causas de diarreia em bezerros (Smith et al., 1989; Santos et al., 2001).

De maneira geral, as infecções por Salmonella resultam em uma variedade de manifestações clínicas que dependem do sorotipo envolvido e da idade do animal (Sarwari et al., 2001). Em bezerros com até seis semanas de idade, os principais sinais clínicos de salmonelose incluem diarreia, febre, desidratação, alterações respiratórias, bacteremia e morte (Wray e Davies, 2000; Veling et al., 2002).

Mas, apesar de $S$. Dublin ser o sorotipo adaptado à espécie bovina (Smith et al., 1989), a maior parte dos estudos sobre as alterações hematológicas e bioquímicas em bezerros induzidas pela salmonelose experimental utiliza o sorotipo $S$. Typhimurium (Smith et al., 1979; Wray, 1980; Santos et al., 2002). Assim, o objetivo do presente estudo foi avaliar as principais alterações bioquímicas, hematológicas e hemogasométricas provocadas pela infecção experimental de bezerros com Salmonella Dublin.

\section{MATERIAL E MÉTODOS}

Foram examinadas amostras de sangue de 12 bezerros machos da raça Holandesa, sadios, com 10 a 15 dias de idade e peso médio de $40,2 \mathrm{~kg}$. Os bezerros foram alojados em abrigos individuais e receberam quatro litros de leite pasteurizado, duas vezes ao dia, além de ração, feno e água à vontade. O estudo foi aprovado pela Comissão de Ética e Bem-Estar Animal da FCAV/UNESP/Campus de Jaboticabal (Protocolo $\mathrm{n}^{\circ}$ 013597-06). Os animais foram distribuídos aleatoriamente em dois grupos experimentais, constituídos por seis animais cada, submetidos aos seguintes tratamentos: grupo-controle (grupo 1): administração oral de $10 \mathrm{~mL}$ de Brain Heart Infusion (caldo BHI; CM0225, Oxoid); e grupo infectado (grupo 2): administração oral de $10^{8}$ UFC de Salmonella Dublin suspensas em $10 \mathrm{~mL}$ de caldo BHI, antes da primeira mamada do dia. O inóculo para a indução da infecção experimental foi preparado a partir de uma amostra de Salmonella Dublin (registro IOC 3101/03) cedida pela Fundação Oswaldo Cruz (Manguinhos-RJ), naturalmente resistente ao ácido nalidíxico.
As amostras de sangue de cada bezerro foram obtidas mediante punção da veia jugular, em oito momentos: minutos antes da inoculação experimental (0h) e 24, 48, 72, 96, 120, 144 e 168 h após a inoculação, utilizando-se o sistema de coleta a vácuo (Vacutainer, Becton Dickinson), em frascos de $10 \mathrm{~mL}$ sem anticoagulante, para realização das análises bioquímicas, frascos de $5 \mathrm{~mL}$ contendo ácido etilenodiaminotetracético tripotássico ( $\mathrm{K}_{3}$ EDTA), para realização do hemograma, frascos de $5 \mathrm{~mL}$ contendo fluoreto de sódio/ácido etilenodiaminotetracético dissódico ( $\mathrm{NaF} / \mathrm{Na}_{2} \mathrm{EDTA}$ ), para dosagem de glicose, e seringas plásticas de $1 \mathrm{~mL}$ contendo heparina de lítio, para hemogasometria e dosagem de eletrólitos. As amostras de sangue dos frascos sem anticoagulante foram centrifugadas a 1.000 $\mathrm{x}$ g durante 10 minutos, logo após a retração do coágulo, enquanto as amostras de sangue de frascos com anticoagulante foram centrifugadas a $1.000 \mathrm{x}$ g durante cinco minutos, obtendo-se o soro e o plasma, respectivamente, necessários para as análises laboratoriais. As amostras de sangue, inclusive aquelas para as análises hemogasométricas, foram mantidas sob refrigeração até a chegada no laboratório. Foram avaliadas as atividades séricas das enzimas aspartato aminotransferase (método cinética UVIFCC), fosfatase alcalina (método de Bowers e McComb modificado), creatina cinase (método IFCC), gamaglutamiltransferase (método de Szasz modificado) e lactato desidrogenase (método do piruvato-lactato) e as concentrações de bilirrubina total e direta (método de SimsHorn), cálcio total (método CPC), ferro (método de Goodwin modificado), fósforo (método de Daly e Ertingshausen modificado), magnésio (método Labtest), proteínas totais (método do biureto), albumina (método verde de bromocresol), creatinina (método Labtest), ureia (método enzimático UV) e glicose (método de GOD-Trinder). Os testes foram realizados utilizando-se conjuntos de reagentes de uso comercial (Labtest, Labtest Diagnóstica), e as leituras dos parâmetros bioquímicos foram realizadas em espectrofotômetro semiautomático (Labquest, Labtest Diagnóstica), em comprimentos de onda específicos para os diversos componentes do sangue. $\mathrm{O}$ teor plasmático de fibrinogênio foi obtido pelo método de precipitação pelo calor e pela leitura em refratômetro (Millar et al., 1971). 
As contagens de hemácias e de leucócitos e o teor de hemoglobina foram obtidos em hemocitômetro semiautomático (CC-530, CELM), e as contagens de plaquetas realizadas em câmara de Neubauer. O volume globular foi obtido pela técnica do microematócrito. A contagem diferencial dos leucócitos foi verificada em esfregaços sanguíneos corados (Thrall, 2007). As variáveis hemogasométricas $\mathrm{pH}$, pressão parcial de oxigênio $\left(\mathrm{prpO}_{2}\right)$, pressão parcial de dióxido de carbono $\left(\mathrm{prpCO}_{2}\right)$, bicarbonato $\left(\mathrm{HCO}_{3}\right)$, dióxido de carbono total $\left(\right.$ totCO $\left.\mathrm{C}_{2}\right)$, saturação de oxigênio $\left(\mathrm{SatO}_{2}\right)$ e excesso de base (excB) e os teores sanguíneos de sódio $(\mathrm{Na})$, potássio $(\mathrm{K})$, cálcio ionizado $\left(\mathrm{Ca}_{\mathrm{i}}\right)$ e cloretos $(\mathrm{Cl})$ foram mensurados em analisador automático (Omni C, Roche). Antes de cada coleta, os bezerros eram submetidos a exames físicos, diariamente (Feitosa, 2004).

O isolamento de Salmonella Dublin nas fezes dos bezerros foi realizado por meio de cultura bacteriológica das amostras de suabes retais, segundo as recomendações de Rice et al. (1997) e Santos et al. (2002), nos mesmos momentos descritos anteriormente para a colheita das amostras de sangue.

Os resultados das análises bioquímicas, hematológicas e hemogasométricas foram submetidos à análise de variância e ao teste de Tukey para comparação das médias, ao nível de significância de 5\% (Zar, 1999).

\section{RESULTADOS}

Entre 24 e 72 horas após a infecção experimental, os animais inoculados com $10^{8}$ UFC de Salmonella Dublin apresentaram sinais de diarreia, febre, desidratação e sintomas respiratórios, como tosse, secreção nasal e taquipneia. Não se constatou alteração no exame físico dos animais do grupo-controle. $\mathrm{O}$ isolamento de Salmonella Dublin em suabes retais ocorreu 24 horas após a infecção experimental, em todos os bezerros inoculados, e não houve isolamento de Salmonella Dublin em nenhuma das amostras de suabes retais dos animais do grupo-controle.

As principais alterações bioquímicas verificadas nos bezerros do grupo infectado, em relação aos do grupo-controle, foram aumento do teor plasmático de fibrinogênio e diminuição das atividades séricas das enzimas ALP e CK e da concentração sérica de albumina (Tab. 1). Também, ao longo do período experimental, foram notadas reduções nas concentrações de glicose e de proteína total nos bezerros do grupo infectado. $\mathrm{O}$ aumento da concentração de fibrinogênio, importante indicador da resposta de fase aguda em bovinos (Godson et al., 1995), é compatível com o quadro inflamatório que acompanha a infecção bacteriana. Os decréscimos nas concentrações de proteína total e albumina provavelmente estão relacionados à perda de proteínas entéricas devido à enterite fibrinopurulenta desenvolvida após a infecção com S. Dublin (Santos et al., 2002). A diminuição da concentração de ferro pode estar relacionada à resposta imune do hospedeiro, mediada pela interleucina-1, que promove redução da concentração desse mineral após a invasão bacteriana, pois o crescimento e a multiplicação das bactérias dependem da disponibilidade de ferro (Kaneko et al., 2008). A redução da concentração de magnésio, verificada em animais com diarreia (Slanina et al., 1984), também pode estar relacionada à maior perda desse componente nas fezes. A diminuição da glicemia pode ser explicada pela inapetência dos bezerros e pela própria multiplicação bacteriana. As variações constatadas nas atividades séricas das enzimas ALP e CK e nas concentrações de bilirrubina total podem estar mais associadas às variações individuais do que à própria infecção experimental.

Apesar de não ter sido constatada diferença nos parâmetros hematológicos nos bezerros dos dois grupos experimentais, notou-se leucocitose nos animais do grupo 2, a partir de 24 horas após a infecção experimental com Salmonella Dublin (Tab. 2), em resposta à infecção bacteriana.

Com relação às variáveis hemogasométricas, verificou-se redução da concentração sanguínea de sódio às 120 e 144 horas após a inoculação, nos bezerros do grupo 2 (Tab. 3), aspecto atribuído à perda desse eletrólito nas fezes (Walker et al., 1998; Kaneko et al., 2008). Também foram verificadas ligeiras diminuições do $\mathrm{pH}$, do exB e da concentração de $\mathrm{HCO}_{3}$ a partir de 72 horas após a infecção (Tab. 3), compatível com quadro discreto de acidose metabólica. 
Tabela 1. Média \pm desvio-padrão de parâmetros bioquímicos de bezerros do grupo-controle $(\mathrm{G} 1)$ e de bezerros infectados experimentalmente com $10^{8}$ UFC de Salmonella Dublin (G2) antes da inoculação (Oh) e $24,48,72,96,120,144$ e $168 \mathrm{~h}$ após a infecção experimental

\begin{tabular}{|c|c|c|c|c|c|c|c|c|}
\hline \multirow[t]{2}{*}{ Grupo } & \multicolumn{8}{|c|}{ Momento após a inoculação (h) } \\
\hline & 0 & 24 & 48 & 72 & 96 & 120 & 144 & 168 \\
\hline \multicolumn{9}{|c|}{ Aspartato aminotransferase (U/L) } \\
\hline G1 & $36,7 \pm 8,76 \mathrm{Aa}$ & $39,3 \pm 4,38 \mathrm{Aa}$ & $36,7 \pm 4,68 \mathrm{Aa}$ & $32,3 \pm 12,1 \mathrm{Aa}$ & $33,2 \pm 13,5 \mathrm{Aa}$ & $33,2 \pm 10,8 \mathrm{Aa}$ & $34,9 \pm 8,55 \mathrm{Aa}$ & $35,8 \pm 9,02 \mathrm{Aa}$ \\
\hline G2 & $36,7 \pm 4,68 \mathrm{Aa}$ & $38,4 \pm 5,42 \mathrm{Aa}$ & $36,7 \pm 5,74 \mathrm{Aa}$ & $36,7 \pm 8,76 \mathrm{Aa}$ & $32,5 \pm 7,77 \mathrm{Aa}$ & $36,7 \pm 12,3 \mathrm{Aa}$ & $38,8 \pm 19,5 \mathrm{Aa}$ & $30,1 \pm 2,62 \mathrm{Aa}$ \\
\hline \multicolumn{9}{|c|}{ Fosfatase alcalina $(\mathrm{U} / \mathrm{L})$} \\
\hline G1 & $135 \pm 22,0 \mathrm{Aa}$ & $145 \pm 30,0 \mathrm{Aa}$ & $159 \pm 48,2 \mathrm{Aa}$ & $167 \pm 58,5 \mathrm{Aa}$ & $163 \pm 49,9 \mathrm{Aa}$ & $171 \pm 63,1 \mathrm{Aa}$ & $169 \pm 68,9 \mathrm{Aa}$ & $169 \pm 65,6 \mathrm{Aa}$ \\
\hline $\mathrm{G} 2$ & $122 \pm 57,4 \mathrm{Aa}$ & $119 \pm 54,9 \mathrm{Aa}$ & $115 \pm 43,4 \mathrm{Aa}$ & $113 \pm 35,0 \mathrm{Ba}$ & $115 \pm 29,6 \mathrm{Ba}$ & $99,5 \pm 24,2 \mathrm{Ba}$ & $91,2 \pm 20,3 \mathrm{Ba}$ & $76,7 \pm 14,2 \mathrm{Ba}$ \\
\hline \multicolumn{9}{|c|}{ Gamaglutamiltransferase (U/L) } \\
\hline G1 & $88,9 \pm 35,4 \mathrm{Aabc}$ & $92,1 \pm 37,7 \mathrm{Aa}$ & $79,4 \pm 34,3 \mathrm{Aad}$ & $77,3 \pm 33,4$ Aad & $74,1 \pm 25,4$ Acd & $72,0 \pm 24,6 \mathrm{Abd}$ & $69,8 \pm 23,3 \mathrm{Ad}$ & $64,5 \pm 22,8 \mathrm{Ad}$ \\
\hline G2 & $98,6 \pm 29,5 \mathrm{Aa}$ & $95,4 \pm 24,5 \mathrm{Aa}$ & $83,8 \pm 19,5 \mathrm{Aac}$ & $75,3 \pm 18,2 \mathrm{Abcd}$ & $71,3 \pm 11,4$ Ade & $66,2 \pm 11,6 \mathrm{Abe}$ & $59,8 \pm 7,25 \mathrm{Ae}$ & $57,3 \pm 9,00 \mathrm{Ae}$ \\
\hline \multicolumn{9}{|c|}{ Lactato desigrogenase (U/L) } \\
\hline G1 & $1.201 \pm 284 \mathrm{Aa}$ & $1.325 \pm 342 \mathrm{Aa}$ & $1.344 \pm 345 \mathrm{Aa}$ & $1.395 \pm 315 \mathrm{Aa}$ & $1.406 \pm 322 \mathrm{Aa}$ & $1.371 \pm 272 \mathrm{Aa}$ & $1.392 \pm 301 \mathrm{Aa}$ & $1.425 \pm 314 \mathrm{Aa}$ \\
\hline $\mathrm{G} 2$ & $1.174 \pm 118 \mathrm{Aa}$ & $1.211 \pm 112 \mathrm{Aa}$ & $1.230 \pm 115 \mathrm{Aa}$ & $1.279 \pm 229 \mathrm{Aa}$ & $1.169 \pm 148 \mathrm{Aa}$ & $1.243 \pm 197 \mathrm{Aa}$ & $1.240 \pm 182 \mathrm{Aa}$ & $1.206 \pm 127 \mathrm{Aa}$ \\
\hline \multicolumn{9}{|c|}{ Creatina cinase (U/L) } \\
\hline G1 & $162 \pm 29,4 \mathrm{Aa}$ & $162 \pm 19,8 \mathrm{Aa}$ & $142 \pm 28,4 \mathrm{Aa}$ & $162 \pm 39,7 \mathrm{Aa}$ & $125 \pm 9,92 \mathrm{Aa}$ & $130 \pm 19,8 \mathrm{Aa}$ & $121 \pm 26,6 \mathrm{Aa}$ & $138 \pm 36,6 \mathrm{Aa}$ \\
\hline G2 & $113 \pm 39,7 \mathrm{Aa}$ & $117 \pm 51,9 \mathrm{Aa}$ & $109 \pm 39,9 \mathrm{Aa}$ & $97,1 \pm 34,4 \mathrm{Aa}$ & $126 \pm 20,3 \mathrm{Aa}$ & $121 \pm 24,3 \mathrm{Aa}$ & $117 \pm 71,7 \mathrm{Aa}$ & $85,0 \pm 14,0 \mathrm{Bb}$ \\
\hline \multicolumn{9}{|c|}{ Albumina (g/dL) } \\
\hline G1 & $2,35 \pm 0,09 \mathrm{Aa}$ & $2,43 \pm 0,10 \mathrm{Aa}$ & $2,43 \pm 0,12 \mathrm{Aa}$ & $2,44 \pm 0,14 \mathrm{Aa}$ & $2,48 \pm 0,16 \mathrm{Aa}$ & $2,50 \pm 0,11 \mathrm{Aa}$ & $2,50 \pm 0,10 \mathrm{Aa}$ & $2,51 \pm 0,12 \mathrm{Aa}$ \\
\hline G2 & $2,25 \pm 0,13 \mathrm{Aa}$ & $2,26 \pm 0,15 \mathrm{Aa}$ & $2,30 \pm 0,19 \mathrm{Aa}$ & $2,26 \pm 0,23 \mathrm{Aa}$ & $2,26 \pm 0,17 \mathrm{Ba}$ & $2,27 \pm 0,19 \mathrm{Ba}$ & $2,19 \pm 0,18 \mathrm{Ba}$ & $2,25 \pm 0,21 \mathrm{Ba}$ \\
\hline \multicolumn{9}{|c|}{ Bilirrubina direta $(\mathrm{mg} / \mathrm{dL})$} \\
\hline G1 & $0,21 \pm 0,09 \mathrm{Aa}$ & $0,20 \pm 0,09 \mathrm{Aa}$ & $0,17 \pm 0,05 \mathrm{Aa}$ & $0,16 \pm 0,04 \mathrm{Aa}$ & $0,15 \pm 0,06 \mathrm{Aa}$ & $0,15 \pm 0,05 \mathrm{Aa}$ & $0,15 \pm 0,04 \mathrm{Aa}$ & $0,14 \pm 0,04 \mathrm{Aa}$ \\
\hline $\mathrm{G} 2$ & $0,23 \pm 0,04 \mathrm{Aa}$ & $0,22 \pm 0,06 \mathrm{Aa}$ & $0,20 \pm 0,06 \mathrm{Aa}$ & $0,17 \pm 0,07 \mathrm{Aa}$ & $0,19 \pm 0,07 \mathrm{Aa}$ & $0,20 \pm 0,08 \mathrm{Aa}$ & $0,16 \pm 0,07 \mathrm{Aa}$ & $0,18 \pm 0,02 \mathrm{Aa}$ \\
\hline \multicolumn{9}{|c|}{ Bilirrubina total $(\mathrm{mg} / \mathrm{dL})$} \\
\hline G1 & $0,70 \pm 0,13 \mathrm{Aa}$ & $0,74 \pm 0,21 \mathrm{Aa}$ & $0,72 \pm 0,19 \mathrm{Aa}$ & $0,67 \pm 0,16 \mathrm{Aa}$ & $0,66 \pm 0,24 \mathrm{Aa}$ & $0,70 \pm 0,23 \mathrm{Aa}$ & $0,72 \pm 0,18 \mathrm{Aa}$ & $0,72 \pm 0,17 \mathrm{Aa}$ \\
\hline G2 & $0,68 \pm 0,41 \mathrm{Aab}$ & $0,67 \pm 0,43 \mathrm{Aa}$ & $0,61 \pm 0,35 \mathrm{Aa}$ & $0,70 \pm 0,38 \mathrm{Aa}$ & $0,52 \pm 0,08 \mathrm{Aa}$ & $0,61 \pm 0,20 \mathrm{Ab}$ & $0,63 \pm 0,14 \mathrm{Ab}$ & $0,58 \pm 0,05 \mathrm{Aa}$ \\
\hline \multicolumn{9}{|c|}{ Cálcio total $(\mathrm{mg} / \mathrm{dL})$} \\
\hline G1 & $9,56 \pm 0,47 \mathrm{Aa}$ & $10,5 \pm 0,52 \mathrm{Ab}$ & $10,6 \pm 0,74 \mathrm{Ab}$ & $10,4 \pm 0,65 \mathrm{Aab}$ & $10,2 \pm 0,56 \mathrm{Aab}$ & $10,3 \pm 0,40 \mathrm{Aab}$ & $10,1 \pm 0,52 \mathrm{Aab}$ & $10,2 \pm 0,36 \mathrm{Aab}$ \\
\hline G2 & $10,2 \pm 1,05 \mathrm{Aa}$ & $11,2 \pm 0,89 \mathrm{Ab}$ & $10,9 \pm 0,85 \mathrm{Aab}$ & $10,7 \pm 0,94 \mathrm{Aab}$ & $10,4 \pm 0,34 \mathrm{Aa}$ & $10,6 \pm 0,17 \mathrm{Aab}$ & $10,7 \pm 0,34 \mathrm{Aab}$ & $10,2 \pm 0,41 \mathrm{Aa}$ \\
\hline \multicolumn{9}{|c|}{ Creatinina (mg/dL) } \\
\hline G1 & $1,28 \pm 0,15 \mathrm{Aa}$ & $1,31 \pm 0,11 \mathrm{Aa}$ & $1,24 \pm 0,07 \mathrm{Aa}$ & $1,23 \pm 0,12 \mathrm{Aa}$ & $1,25 \pm 0,11 \mathrm{Aa}$ & $1,22 \pm 0,11 \mathrm{Aa}$ & $1,28 \pm 0,14 \mathrm{Aa}$ & $1,29 \pm 0,17 \mathrm{Aa}$ \\
\hline G2 & $1,17 \pm 0,08 \mathrm{Aa}$ & $1,12 \pm 0,08 \mathrm{Aa}$ & $1,08 \pm 0,10 \mathrm{Aa}$ & $1,10 \pm 0,12 \mathrm{Aa}$ & $1,17 \pm 0,12 \mathrm{Aa}$ & $1,16 \pm 0,15 \mathrm{Aa}$ & $1,17 \pm 0,16 \mathrm{Aa}$ & $1,17 \pm 0,12 \mathrm{Aa}$ \\
\hline \multicolumn{9}{|c|}{ Ferro $(\mu \mathrm{g} / \mathrm{dL})$} \\
\hline G1 & $136 \pm 54,8 \mathrm{Aa}$ & $147 \pm 64,7 \mathrm{Aa}$ & $149 \pm 57,2 \mathrm{Aa}$ & $156 \pm 60,4 \mathrm{Aa}$ & $146 \pm 56,7 \mathrm{Aa}$ & $127 \pm 35,4 \mathrm{Aa}$ & $156 \pm 53,9 \mathrm{Aa}$ & $128 \pm 52,4 \mathrm{Aa}$ \\
\hline G2 & $90,5 \pm 19,7 \mathrm{Aa}$ & $96,0 \pm 37,2 \mathrm{Aa}$ & $68,3 \pm 7,37 \mathrm{Aa}$ & $77,3 \pm 9,27 \mathrm{Aa}$ & $106 \pm 68,0 \mathrm{Aa}$ & $90,0 \pm 35,9 \mathrm{Aa}$ & $77,8 \pm 17,2 \mathrm{Ba}$ & $78,5 \pm 19,2 \mathrm{Aa}$ \\
\hline \multicolumn{9}{|c|}{ Fibrinogênio (g/dL) } \\
\hline G1 & $0,77 \pm 0,23 \mathrm{Aa}$ & $0,50 \pm 0,11 \mathrm{Aa}$ & $0,70 \pm 0,24 \mathrm{Aa}$ & $0,70 \pm 0,17 \mathrm{Aa}$ & $0,70 \pm 0,17 \mathrm{Aa}$ & $0,57 \pm 0,08 \mathrm{Aa}$ & $0,63 \pm 0,08 \mathrm{Aa}$ & $0,57 \pm 0,15 \mathrm{Aa}$ \\
\hline G2 & $0,67 \pm 0,24 \mathrm{Aa}$ & $0,77 \pm 0,20 \mathrm{Aa}$ & $0,93 \pm 0,27 \mathrm{Aa}$ & $0,87 \pm 0,33 \mathrm{Aa}$ & $0,96 \pm 0,17 \mathrm{Aa}$ & $0,92 \pm 0,18 \mathrm{Ba}$ & $0,88 \pm 0,23 \mathrm{Aa}$ & $0,95 \pm 0,10 \mathrm{Ba}$ \\
\hline \multicolumn{9}{|c|}{ Fósforo (mg/dL) } \\
\hline G1 & $7,12 \pm 0,42 \mathrm{Aa}$ & $7,63 \pm 0,59 \mathrm{Aa}$ & $7,87 \pm 0,72 \mathrm{Aa}$ & $7,82 \pm 0,59 \mathrm{Aa}$ & $7,90 \pm 0,69 \mathrm{Aa}$ & $7,94 \pm 0,64 \mathrm{Aa}$ & $7,75 \pm 0,51 \mathrm{Aa}$ & $7,82 \pm 0,73 \mathrm{Aa}$ \\
\hline G2 & $7,47 \pm 1,04 \mathrm{Aa}$ & $7,44 \pm 1,26 \mathrm{Aa}$ & $7,32 \pm 0,87 \mathrm{Aa}$ & $7,17 \pm 1,43 \mathrm{Aa}$ & $7,28 \pm 0,91 \mathrm{Aa}$ & $7,36 \pm 0,90 \mathrm{Aa}$ & $7,42 \pm 0,51 \mathrm{Aa}$ & $7,44 \pm 0,76 \mathrm{Aa}$ \\
\hline \multicolumn{9}{|c|}{ Glicose (mg/dL) } \\
\hline G1 & $75,0 \pm 7,71 \mathrm{Aab}$ & $74,7 \pm 8,80 \mathrm{Aab}$ & $71,0 \pm 10,2 \mathrm{Aab}$ & $72,1 \pm 6,83 \mathrm{Aab}$ & $71,0 \pm 7,51 \mathrm{Aab}$ & $75,7 \pm 9,28 \mathrm{Aab}$ & $69,0 \pm 5,77 \mathrm{Ab}$ & $68,6 \pm 10,6 \mathrm{Ab}$ \\
\hline G2 & $70,2 \pm 18,5 \mathrm{Aab}$ & $71,5 \pm 13,3 \mathrm{Aab}$ & $73,5 \pm 14,7 \mathrm{Aab}$ & $72,8 \pm 16,6 \mathrm{Aab}$ & $66,1 \pm 16,9 \mathrm{Ab}$ & $64,5 \pm 13,1 \mathrm{Ab}$ & $61,9 \pm 16,2 \mathrm{Ab}$ & $65,8 \pm 9,61 \mathrm{Ab}$ \\
\hline \multicolumn{9}{|c|}{ Magnésio (mg/dL) } \\
\hline G1 & $1,91 \pm 0,12 \mathrm{Aa}$ & $1,96 \pm 0,19 \mathrm{Aa}$ & $1,95 \pm 0,19 \mathrm{Aa}$ & $1,98 \pm 0,13 \mathrm{Aa}$ & $1,93 \pm 0,12 \mathrm{Aa}$ & $2,02 \pm 0,08 \mathrm{Aa}$ & $2,00 \pm 0,08 \mathrm{Aa}$ & $2,01 \pm 0,07 \mathrm{Aa}$ \\
\hline G2 & $1,86 \pm 0,18 \mathrm{Aa}$ & $1,88 \pm 0,20 \mathrm{Aa}$ & $1,81 \pm 0,23 \mathrm{Aa}$ & $1,79 \pm 0,28 \mathrm{Aa}$ & $1,77 \pm 0,24 \mathrm{Aa}$ & $1,77 \pm 0,19 \mathrm{Aa}$ & $1,75 \pm 0,30 \mathrm{Aa}$ & $1,66 \pm 0,11 \mathrm{Bb}$ \\
\hline \multicolumn{9}{|c|}{ Proteína total $(\mathrm{g} / \mathrm{dL})$} \\
\hline G1 & $6,60 \pm 0,66 \mathrm{Aa}$ & $6,53 \pm 0,63 \mathrm{Aa}$ & $6,54 \pm 0,48 \mathrm{Aa}$ & $6,66 \pm 0,52 \mathrm{Aa}$ & $6,62 \pm 0,41 \mathrm{Aa}$ & $6,63 \pm 0,45 \mathrm{Aa}$ & $6,49 \pm 0,46 \mathrm{Aa}$ & $6,54 \pm 0,39 \mathrm{Aa}$ \\
\hline G2 & $6,56 \pm 0,72 \mathrm{Aab}$ & $6,49 \pm 0,76 \mathrm{Aab}$ & $6,47 \pm 0,48 \mathrm{Aab}$ & $6,44 \pm 0,47 \mathrm{Aab}$ & $6,25 \pm 0,45 \mathrm{Aab}$ & $6,26 \pm 0,49 \mathrm{Aab}$ & $6,09 \pm 0,58 \mathrm{Ab}$ & $6,52 \pm 0,28 \mathrm{Aab}$ \\
\hline & & & & Ureia (mg/c & & & & \\
\hline G1 & $22,2 \pm 2,64 \mathrm{Aa}$ & $23,3 \pm 4,61 \mathrm{Aa}$ & $22,7 \pm 3,73 \mathrm{Aa}$ & $25,3 \pm 3,59 \mathrm{Aa}$ & $23,8 \pm 4,42 \mathrm{Aa}$ & $23,1 \pm 3,80 \mathrm{Aa}$ & $24,2 \pm 3,96 \mathrm{Aa}$ & $23,4 \pm 3,96 \mathrm{Aa}$ \\
\hline G2 & $21,9 \pm 3,70 \mathrm{Aa}$ & $21,8 \pm 9,05 \mathrm{Aa}$ & $24,8 \pm 11,6 \mathrm{Aa}$ & $26,9 \pm 16,5 \mathrm{Aa}$ & $29,0 \pm 18,8 \mathrm{Aa}$ & $30,4 \pm 16,5 \mathrm{Aa}$ & $35,5 \pm 29,9 \mathrm{Aa}$ & $24,1 \pm 7,02 \mathrm{Aa}$ \\
\hline
\end{tabular}

Médias seguidas de letras maiúsculas iguais na mesma coluna e letras minúsculas iguais na mesma linha não diferem entre si pelo teste Tukey $(\mathrm{P}>0,05)$. 
Hemograma e perfil bioquímico...

Tabela 2. Média \pm desvio-padrão de parâmetros hematológicos de bezerros do grupo-controle $(\mathrm{G} 1)$ e bezerros infectados experimentalmente com $10^{8}$ UFC de Salmonella Dublin (G2) antes da inoculação (Oh) e $24,48,72,96,120,144$ e $168 \mathrm{~h}$ após a infecção experimental

\begin{tabular}{|c|c|c|c|c|c|c|c|c|}
\hline \multirow{2}{*}{ Grup } & \multicolumn{8}{|c|}{ Momento após a inoculação (h) } \\
\hline & 0 & 24 & 48 & 72 & 96 & 120 & 144 & 168 \\
\hline \multicolumn{9}{|c|}{ Hemácias $(\times 10 \% / \mu \mathrm{L})$} \\
\hline G1 & $7,82 \pm 1,05 \mathrm{Aa}$ & $7,78 \pm 1,25 \mathrm{Aab}$ & $7,58 \pm 0,85 \mathrm{Aab}$ & $7,76 \pm 0,83 \mathrm{Aab}$ & $7,43 \pm 0,99 \mathrm{Aab}$ & $7,50 \pm 0,71 \mathrm{Aab}$ & $7,38 \pm 0,95 \mathrm{Aab}$ & $6,97 \pm 0,87 \mathrm{Ab}$ \\
\hline $\mathrm{G} 2$ & $7,53 \pm 1,15 \mathrm{Aa}$ & $6,98 \pm 1,11 \mathrm{Aab}$ & $7,18 \pm 0,96 \mathrm{Aab}$ & $7,00 \pm 1,03 \mathrm{Aab}$ & $7,14 \pm 0,95 \mathrm{Aab}$ & $7,17 \pm 1,13 \mathrm{Aab}$ & $6,60 \pm 0,98 \mathrm{Ab}$ & $6,74 \pm 0,37 \mathrm{Ab}$ \\
\hline \multicolumn{9}{|c|}{ 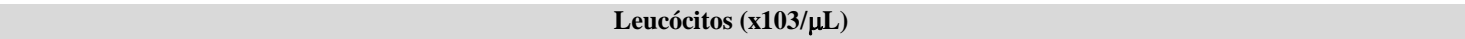 } \\
\hline G1 & $9,90 \pm 5,93 \mathrm{Aa}$ & $10,5 \pm 5,74 \mathrm{Aa}$ & $10,0 \pm 5,88 \mathrm{Aa}$ & $10,3 \pm 4,79 \mathrm{Aa}$ & $9,40 \pm 2,99 \mathrm{Aa}$ & $8,22 \pm 2,30 \mathrm{Aa}$ & $7,48 \pm 2,10 \mathrm{Aa}$ & $8,60 \pm 4,06 \mathrm{Aa}$ \\
\hline $\mathrm{G} 2$ & $12,0 \pm 7,41 \mathrm{Aa}$ & $13,0 \pm 7,16 \mathrm{Aa}$ & $14,7 \pm 7,66 \mathrm{Aa}$ & $16,7 \pm 9,26 \mathrm{Aa}$ & $13,7 \pm 7,94 \mathrm{Aa}$ & $12,5 \pm 4,63 \mathrm{Aa}$ & $12,5 \pm 5,58 \mathrm{Aa}$ & $12,0 \pm 5,94 \mathrm{Aa}$ \\
\hline \multicolumn{9}{|c|}{ Plaquetas $(\mathbf{x 1 0 3 / \mu L )}$} \\
\hline G1 & $473 \pm 30,2 \mathrm{Aa}$ & $476 \pm 57,0 \mathrm{Aa}$ & $480 \pm 72,0 \mathrm{Aa}$ & $447 \pm 57,4 \mathrm{Aa}$ & $415 \pm 36,6 \mathrm{Aa}$ & $443 \pm 48,6 \mathrm{Aa}$ & $469 \pm 29,6 \mathrm{Aa}$ & $452 \pm 48,9 \mathrm{Aa}$ \\
\hline G2 & $486 \pm 105 \mathrm{Aa}$ & $509 \pm 172 \mathrm{Aa}$ & $539 \pm 196 \mathrm{Aa}$ & $519 \pm 182 \mathrm{Aa}$ & $526 \pm 220 \mathrm{Aa}$ & $502 \pm 224 \mathrm{Aa}$ & $551 \pm 274 \mathrm{Aa}$ & $456 \pm 24,6 \mathrm{Aa}$ \\
\hline \multicolumn{9}{|c|}{ Hemoglobina (g/dL) } \\
\hline G1 & $10,8 \pm 1,87 \mathrm{Aa}$ & $10,6 \pm 1,73 \mathrm{Aab}$ & $10,4 \pm 1,65 \mathrm{Aabc}$ & $10,3 \pm 1,49 \mathrm{Aabc}$ & $10,1 \pm 1,35 \mathrm{Aabc}$ & $10,2 \pm 1,39 \mathrm{Aabc}$ & $9,70 \pm 1,29 \mathrm{Abc}$ & $9,56 \pm 1,26 \mathrm{Ac}$ \\
\hline $\mathrm{G} 2$ & $9,87 \pm 0,90 \mathrm{Aa}$ & $9,87 \pm 1,22 \mathrm{Aa}$ & $9,88 \pm 1,32 \mathrm{Aa}$ & $9,92 \pm 1,09 \mathrm{Aa}$ & $9,67 \pm 0,91 \mathrm{Aa}$ & $9,53 \pm 0,88 \mathrm{Aa}$ & $9,33 \pm 0,83 \mathrm{Aa}$ & $9,48 \pm 0,72 \mathrm{Aa}$ \\
\hline \multicolumn{9}{|c|}{ Volume globular (\%) } \\
\hline G1 & $32,5 \pm 5,32 \mathrm{Aa}$ & $32,2 \pm 5,56 \mathrm{Aab}$ & $31,2 \pm 5,38 \mathrm{Aab}$ & $31,0 \pm 4,90 \mathrm{Aab}$ & $30,7 \pm 5,32 \mathrm{Aab}$ & $30,3 \pm 4,32 \mathrm{Aab}$ & $29,2 \pm 4,58 \mathrm{Aab}$ & $28,7 \pm 4,13 \mathrm{Ab}$ \\
\hline $\mathrm{G} 2$ & $29,5 \pm 4,42 \mathrm{Aa}$ & $29,3 \pm 5,01 \mathrm{Aa}$ & $29,3 \pm 4,68 \mathrm{Aa}$ & $28,8 \pm 3,60 \mathrm{Aab}$ & $28,4 \pm 3,44 \mathrm{Aab}$ & $28,6 \pm 4,62 \mathrm{Aab}$ & $27,8 \pm 3,96 \mathrm{Aab}$ & $26,7 \pm 2,50 \mathrm{Ab}$ \\
\hline \multicolumn{9}{|c|}{ Basófilos $(/ \mu \mathrm{L})$} \\
\hline G1 & $0,0 \pm 0,0 \mathrm{Aa}$ & $0,0 \pm 0,0 \mathrm{Aa}$ & $0,0 \pm 0,0 \mathrm{Aa}$ & $33,0 \pm 80,8 \mathrm{Aa}$ & $0,0 \pm 0,0 \mathrm{Aa}$ & $13,3 \pm 32,7 \mathrm{Aa}$ & $0,0 \pm 0,0 \mathrm{Aa}$ & $0,0 \pm 0,0 \mathrm{Aa}$ \\
\hline $\mathrm{G} 2$ & $11,2 \pm 27,3 \mathrm{Aa}$ & $0,0 \pm 0,0 \mathrm{Aa}$ & $0,0 \pm 0,0 \mathrm{Aa}$ & $0,0 \pm 0,0 \mathrm{Aa}$ & $0,0 \pm 0,0 \mathrm{Aa}$ & $0,0 \pm 0,0 \mathrm{Aa}$ & $0,0 \pm 0,0 \mathrm{Aa}$ & $0,0 \pm 0,0 \mathrm{Aa}$ \\
\hline \multicolumn{9}{|c|}{ Eosinófilos $(/ \mu \mathrm{L})$} \\
\hline G1 & $53,3 \pm 43,8 \mathrm{Aa}$ & $77,2 \pm 79,69 \mathrm{Aa}$ & $49,3 \pm 64,7 \mathrm{Aa}$ & $78,0 \pm 73,6$ Aa & $37,5 \pm 66,6 \mathrm{Aa}$ & $26,7 \pm 65,3 \mathrm{Aa}$ & $38,0 \pm 42,4 \mathrm{Aa}$ & $74,5 \pm 86,0 \mathrm{Aa}$ \\
\hline G2 & $0,0 \pm 0,0 \mathrm{Aa}$ & $45,3 \pm 111 \mathrm{Aa}$ & $0,0 \pm 0,0 \mathrm{Aa}$ & $65,0 \pm 112 \mathrm{Aa}$ & $0,0 \pm 0,0 \mathrm{Aa}$ & $0,0 \pm 0,0 \mathrm{Aa}$ & $0,0 \pm 0,0 \mathrm{Aa}$ & $18,0 \pm 36,0 \mathrm{Aa}$ \\
\hline \multicolumn{9}{|c|}{ Neutrófilos bastonetes $(/ \mu \mathrm{L})$} \\
\hline G1 & $253 \pm 138 \mathrm{Aa}$ & $165 \pm 103 \mathrm{Aa}$ & $155 \pm 72,8 \mathrm{Aa}$ & $121 \pm 111 \mathrm{Aa}$ & $277 \pm 141 \mathrm{Aa}$ & $216 \pm 159 \mathrm{Aa}$ & $230 \pm 112 \mathrm{Aa}$ & $242 \pm 160 \mathrm{Aa}$ \\
\hline $\mathrm{G} 2$ & $211 \pm 334 \mathrm{Aa}$ & $321 \pm 332 \mathrm{Aa}$ & $1.123 \pm 2.185 \mathrm{Aa}$ & $2.691 \pm 5.086 \mathrm{Aa}$ & $476 \pm 675 \mathrm{Aa}$ & $534 \pm 690 \mathrm{Aa}$ & $1.162 \pm 1.822 \mathrm{Aa}$ & $446 \pm 542 \mathrm{Aa}$ \\
\hline \multicolumn{9}{|c|}{ Neutrófilos segmentados $(/ \mu \mathrm{L})$} \\
\hline G1 & $4.611 \pm 4.892 \mathrm{Aa}$ & $4.236 \pm 4.538 \mathrm{Aa}$ & $4.375 \pm 4.756 \mathrm{Aa}$ & $4.607 \pm 4.860 \mathrm{Aa}$ & $3.313 \pm 2.400 \mathrm{Aa}$ & $2.204 \pm 924 \mathrm{Aa}$ & $1.814 \pm 766 \mathrm{Aa}$ & $2.445 \pm 2.621 \mathrm{Aa}$ \\
\hline $\mathrm{G} 2$ & $5.260 \pm 2.083 \mathrm{Aa}$ & $5.921 \pm 1.695 \mathrm{Aa}$ & $6.497 \pm 3.125 \mathrm{Aa}$ & $7.214 \pm 2.684 \mathrm{Aa}$ & $6.742 \pm 4.232 \mathrm{Aa}$ & $5.383 \pm 1.856 \mathrm{Aa}$ & $4.220 \pm 1.726 \mathrm{Aa}$ & $4.566 \pm 2.828 \mathrm{Aa}$ \\
\hline \multicolumn{9}{|c|}{ Linfócitos $(/ \mu \mathrm{L})$} \\
\hline G1 & $4.806 \pm 1.936 \mathrm{Aa}$ & $5.707 \pm 2.168 \mathrm{Aa}$ & $5.306 \pm 2.488 \mathrm{Aa}$ & $5.342 \pm 2.209 \mathrm{Aa}$ & $5.552 \pm 1.714 \mathrm{Aa}$ & $5.519 \pm 2.387 \mathrm{Aa}$ & $5.280 \pm 1.893 \mathrm{Aa}$ & $5.664 \pm 2.080 \mathrm{Aa}$ \\
\hline $\mathrm{G} 2$ & $6.404 \pm 5.411 \mathrm{Aa}$ & $6.681 \pm 5.776 \mathrm{Aa}$ & $6.891 \pm 4.969 \mathrm{Aa}$ & $6.481 \pm 5.724 \mathrm{Aa}$ & $6.305 \pm 3.806 \mathrm{Aa}$ & $6.377 \pm 5.384 \mathrm{Aa}$ & $6.834 \pm 4.438 \mathrm{Aa}$ & $6.688 \pm 2.573 \mathrm{Aa}$ \\
\hline \multicolumn{9}{|c|}{ Monócitos $(/ \mu \mathbf{L})$} \\
\hline G1 & $176 \pm 82,1 \mathrm{Aa}$ & $315 \pm 379 \mathrm{Aa}$ & $164 \pm 108 \mathrm{Aa}$ & $119 \pm 59,68 \mathrm{Aa}$ & $220 \pm 88,10 \mathrm{Aa}$ & $238 \pm 84,10 \mathrm{Aa}$ & $121 \pm 100 \mathrm{Aa}$ & $175 \pm 151 \mathrm{Aa}$ \\
\hline $\mathrm{G} 2$ & $147 \pm 133 \mathrm{Aa}$ & $81,8 \pm 107 \mathrm{Aa}$ & $168 \pm 179 \mathrm{Aa}$ & $265 \pm 178 \mathrm{Aa}$ & $177 \pm 172 \mathrm{Aa}$ & $166 \pm 258 \mathrm{Aa}$ & $247 \pm 384 \mathrm{Aa}$ & $331 \pm 249 \mathrm{Aa}$ \\
\hline
\end{tabular}

Médias seguidas de letras maiúsculas iguais na mesma coluna e letras minúsculas iguais na mesma linha não diferem entre si pelo teste Tukey $(\mathrm{P}>0,05)$. 
Tabela 3. Média \pm desvio-padrão de parâmetros hemogasométricos e da concentração sangüínea de eletrólitos de bezerros do grupo-controle $(\mathrm{G} 1)$ e bezerros infectados experimentalmente com $10^{8}$ UFC de Salmonella Dublin (G2) antes da inoculação (0h) e 24, 48, 72, 96, 120, 144 e 168h após a inoculação

\begin{tabular}{|c|c|c|c|c|c|c|c|c|}
\hline \multirow{2}{*}{ Grupo } & \multicolumn{8}{|c|}{ Momento após a inoculação (h) } \\
\hline & 0 & 24 & 48 & 72 & 96 & 120 & 144 & 168 \\
\hline \multicolumn{9}{|c|}{ pH } \\
\hline G1 & $7,35 \pm 0,03 \mathrm{Aa}$ & $7,34 \pm 0,03 \mathrm{Aa}$ & $7,34 \pm 0,01 \mathrm{Aa}$ & $7,34 \pm 0,01 \mathrm{Aa}$ & $7,36 \pm 0,04 \mathrm{Aa}$ & $7,36 \pm 0,02 \mathrm{Aa}$ & $7,37 \pm 0,02 \mathrm{Aa}$ & $7,37 \pm 0,02 \mathrm{Aa}$ \\
\hline G2 & $7,33 \pm 0,02 \mathrm{Aa}$ & $7,35 \pm 0,02 \mathrm{Aa}$ & $7,36 \pm 0,02 \mathrm{Aa}$ & $7,32 \pm 0,02 \mathrm{Aa}$ & $7,31 \pm 0,03 \mathrm{Aa}$ & $7,31 \pm 0,03 \mathrm{Aa}$ & $7,28 \pm 0,03 \mathrm{Aa}$ & $7,35 \pm 0,03 \mathrm{Aa}$ \\
\hline \multicolumn{9}{|c|}{$\mathrm{HCO3}(\mathrm{mmol} / \mathrm{L})$} \\
\hline G1 & $24,7 \pm 1,77 \mathrm{Aa}$ & $26,1 \pm 1,37 \mathrm{Aa}$ & $25,7 \pm 1,33 \mathrm{Aa}$ & $27,0 \pm 1,86 \mathrm{Aa}$ & $27,0 \pm 0,82 \mathrm{Aa}$ & $27,9 \pm 1,34 \mathrm{Aa}$ & $27,9 \pm 0,63 \mathrm{Aa}$ & $28,6 \pm 1,83 \mathrm{Aa}$ \\
\hline $\mathrm{G} 2$ & $26,5 \pm 2,98 \mathrm{Aa}$ & $28,0 \pm 2,54 \mathrm{Aa}$ & $26,6 \pm 3,20 \mathrm{Aa}$ & $24,8 \pm 6,97 \mathrm{Aa}$ & $23,6 \pm 8,63 \mathrm{Aa}$ & $23,3 \pm 8,92 \mathrm{Aa}$ & $22,4 \pm 8,83 \mathrm{Aa}$ & $26,9 \pm 1,59 \mathrm{Aa}$ \\
\hline \multicolumn{9}{|c|}{ Excesso de base $(\mathrm{mmol} / \mathrm{L})$} \\
\hline G1 & $-0,72 \pm 1,95 \mathrm{Aa}$ & $0,28 \pm 0,94 \mathrm{Aa}$ & $-0,02 \pm 0,96 \mathrm{Aa}$ & $1,08 \pm 1,66 \mathrm{Aa}$ & $1,40 \pm 0,88 \mathrm{Aa}$ & $2,22 \pm 1,30 \mathrm{Aa}$ & $2,40 \pm 0,36 \mathrm{Aa}$ & $3,58 \pm 2,35 \mathrm{Aa}$ \\
\hline $\mathrm{G} 2$ & $0,80 \pm 3,33 \mathrm{Aa}$ & $2,48 \pm 2,96 \mathrm{Aa}$ & $1,40 \pm 2,88 \mathrm{Aa}$ & $-0,85 \pm 7,42 \mathrm{Aa}$ & $-1,68 \pm 9,67 \mathrm{Aa}$ & $-2,14 \pm 9,97 \mathrm{Aa}$ & $\stackrel{-}{3,46 \pm 11,04 \mathrm{Aa}}$ & $-2,05 \pm 1,26 \mathrm{Aa}$ \\
\hline \multicolumn{9}{|c|}{$\mathrm{CO} 2$ total $(\mathrm{mmol} / \mathrm{L})$} \\
\hline G1 & $22,7 \pm 1,30 \mathrm{Aa}$ & $24,3 \pm 1,28 \mathrm{Aa}$ & $23,9 \pm 1,03 \mathrm{Aa}$ & $25,1 \pm 1,52 \mathrm{Aa}$ & $25,2 \pm 0,77 \mathrm{Aa}$ & $26,0 \pm 1,69 \mathrm{Aa}$ & $25,8 \pm 1,29 \mathrm{Aa}$ & $26,6 \pm 1,71 \mathrm{Aa}$ \\
\hline G2 & $23,8 \pm 4,29 \mathrm{Aa}$ & $25,4 \pm 4,00 \mathrm{Aa}$ & $24,3 \pm 2,84 \mathrm{Aa}$ & $22,2 \pm 6,07 \mathrm{Aa}$ & $22,8 \pm 8,34 \mathrm{Aa}$ & $22,9 \pm 8,94 \mathrm{Aa}$ & $20,9 \pm 7,98 \mathrm{Aa}$ & $23,8 \pm 2,60 \mathrm{Aa}$ \\
\hline \multicolumn{9}{|c|}{ Pressão parcial de $\mathrm{O} 2(\mathrm{mmHg})$} \\
\hline G1 & $38,5 \pm 5,17 \mathrm{Aa}$ & $36,4 \pm 7,59 \mathrm{Aa}$ & $38,4 \pm 7,44 \mathrm{Aa}$ & $35,9 \pm 5,88 \mathrm{Aa}$ & $39,1 \pm 5,87 \mathrm{Aa}$ & $33,1 \pm 4,33 \mathrm{Aa}$ & $36,3 \pm 5,50 \mathrm{Aa}$ & $39,2 \pm 9,26 \mathrm{Aa}$ \\
\hline $\mathrm{G} 2$ & $36,9 \pm 8,29 \mathrm{Aa}$ & $36,0 \pm 4,31 \mathrm{Aa}$ & $39,8 \pm 3,12 \mathrm{Aa}$ & $39,9 \pm 5,64 \mathrm{Aa}$ & $41,6 \pm 4,03 \mathrm{Aa}$ & $42,1 \pm 3,01 \mathrm{Aa}$ & $40,9 \pm 5,61 \mathrm{Aa}$ & $36,3 \pm 6,63 \mathrm{Aa}$ \\
\hline \multicolumn{9}{|c|}{ Pressão parcial de CO2 (mmHg) } \\
\hline G1 & $46,6 \pm 2,91 \mathrm{Aa}$ & $50,2 \pm 4,88 \mathrm{Aa}$ & $49,1 \pm 3,42 \mathrm{Aa}$ & $51,6 \pm 3,63 \mathrm{Aa}$ & $50,0 \pm 5,06 \mathrm{Aa}$ & $51,1 \pm 2,92 \mathrm{Aa}$ & $50,6 \pm 3,25 \mathrm{Aa}$ & $48,4 \pm 5,15 \mathrm{Aa}$ \\
\hline $\mathrm{G} 2$ & $49,5 \pm 3,88 \mathrm{Aa}$ & $49,8 \pm 6,21 \mathrm{Aa}$ & $50,3 \pm 5,92 \mathrm{Aa}$ & $50,3 \pm 10,3 \mathrm{Aa}$ & $46,0 \pm 11,0 \mathrm{Aa}$ & $44,0 \pm 11,2 \mathrm{Aa}$ & $44,2 \pm 9,16 \mathrm{Aa}$ & $50,7 \pm 5,95 \mathrm{Aa}$ \\
\hline \multicolumn{9}{|c|}{ Saturação de $02(\%)$} \\
\hline G1 & $60,2 \pm 4,80 \mathrm{Aa}$ & $53,9 \pm 11,5 \mathrm{Aa}$ & $57,4 \pm 7,65 \mathrm{Aa}$ & $57,5 \pm 8,52 \mathrm{Aa}$ & $57,0 \pm 10,3 \mathrm{Aa}$ & $52,6 \pm 8,05 \mathrm{Aa}$ & $57,7 \pm 9,40 \mathrm{Aa}$ & $62,7 \pm 14,6$ Aa \\
\hline G2 & $55,0 \pm 12,9 \mathrm{Aa}$ & $58,1 \pm 5,91 \mathrm{Aa}$ & $61,9 \pm 4,87 \mathrm{Aa}$ & $59,2 \pm 6,23 \mathrm{Aa}$ & $61,0 \pm 2,84 \mathrm{Aa}$ & $62,6 \pm 8,31 \mathrm{Aa}$ & $64,1 \pm 8,22 \mathrm{Aa}$ & $54,5 \pm 15,4 \mathrm{Aa}$ \\
\hline \multicolumn{9}{|c|}{$\mathrm{Na}(\mathrm{mmol} / \mathrm{L})$} \\
\hline G1 & $143 \pm 2,05 \mathrm{Aa}$ & $143 \pm 1,22 \mathrm{Aa}$ & $142 \pm 1,46 \mathrm{Aa}$ & $141 \pm 0,70 \mathrm{Aa}$ & $143 \pm 1,89 \mathrm{Aa}$ & $143 \pm 1,23 \mathrm{Aa}$ & $143 \pm 0,67 \mathrm{Aa}$ & $143 \pm 1,33 \mathrm{Aa}$ \\
\hline $\mathrm{G} 2$ & $142 \pm 3,26 \mathrm{Aa}$ & $141 \pm 2,23 \mathrm{Aab}$ & $140 \pm 2,24 \mathrm{Aab}$ & $140 \pm 3,63 \mathrm{Aab}$ & $139 \pm 4,62 \mathrm{Aab}$ & $138 \pm 5,03 \mathrm{Bb}$ & $139 \pm 5,55 \mathrm{Bb}$ & $141 \pm 2,92 \mathrm{Aab}$ \\
\hline \multicolumn{9}{|c|}{ K $(\mathbf{m m o l} / \mathbf{L})$} \\
\hline G1 & $4,11 \pm 0,35 \mathrm{Aa}$ & $3,98 \pm 0,36 \mathrm{Aa}$ & $4,01 \pm 0,39 \mathrm{Aa}$ & $3,97 \pm 0,19 \mathrm{Aa}$ & $3,82 \pm 0,18 \mathrm{Aa}$ & $3,87 \pm 0,19 \mathrm{Aa}$ & $3,96 \pm 0,31 \mathrm{Aa}$ & $4,00 \pm 0,22 \mathrm{Aa}$ \\
\hline G2 & $3,76 \pm 0,15 \mathrm{Aa}$ & $3,75 \pm 0,15 \mathrm{Aa}$ & $3,76 \pm 0,05 \mathrm{Aa}$ & $3,65 \pm 0,21 \mathrm{Aa}$ & $3,57 \pm 0,16$ Aa & $3,68 \pm 0,18 \mathrm{Aa}$ & $3,78 \pm 0,12 \mathrm{Aa}$ & $3,94 \pm 0,27 \mathrm{Aa}$ \\
\hline \multicolumn{9}{|c|}{$\mathrm{Cl}(\mathrm{mmol} / \mathrm{L})$} \\
\hline G1 & $107 \pm 0,80 \mathrm{Aa}$ & $105 \pm 1,52 \mathrm{Aa}$ & $104 \pm 1,12 \mathrm{Aa}$ & $103 \pm 0,60 \mathrm{Aa}$ & $105 \pm 2,09 \mathrm{Aa}$ & $104 \pm 0,83 \mathrm{Aa}$ & $103 \pm 1,17 \mathrm{Aa}$ & $105 \pm 0,93 \mathrm{Aa}$ \\
\hline $\mathrm{G} 2$ & $105 \pm 4,08 \mathrm{Aa}$ & $104 \pm 4,06 \mathrm{Aa}$ & $103 \pm 2,62 \mathrm{Aa}$ & $103 \pm 2,62 \mathrm{Aa}$ & $104 \pm 1,53 \mathrm{Aa}$ & $102 \pm 2,49 \mathrm{Aa}$ & $103 \pm 1,75 \mathrm{Aa}$ & $102 \pm 2,37 \mathrm{Aa}$ \\
\hline \multicolumn{9}{|c|}{$\mathrm{Ca}_{\mathbf{i}}(\mathbf{m m o l} / \mathrm{L})$} \\
\hline G1 & $0,71 \pm 0,08 \mathrm{Aa}$ & $0,76 \pm 0,06 \mathrm{Aa}$ & $0,78 \pm 0,05 \mathrm{Aa}$ & $0,79 \pm 0,07 \mathrm{Aa}$ & $0,71 \pm 0,09 \mathrm{Aa}$ & $0,73 \pm 0,03 \mathrm{Aa}$ & $0,74 \pm 0,08 \mathrm{Aa}$ & $0,66 \pm 0,07 \mathrm{Aa}$ \\
\hline G2 & $0,66 \pm 0,05 \mathrm{Aa}$ & $0,69 \pm 0,10 \mathrm{Aa}$ & $0,80 \pm 0,10 \mathrm{Aa}$ & $0,67 \pm 0,12 \mathrm{Aa}$ & $0,67 \pm 0,03 \mathrm{Aa}$ & $0,75 \pm 0,09 \mathrm{Aa}$ & $0,75 \pm 0,10 \mathrm{Aa}$ & $0,73 \pm 0,04 \mathrm{Aa}$ \\
\hline
\end{tabular}

Médias seguidas de letras maiúsculas iguais na mesma coluna e letras minúsculas iguais na mesma linha não diferem entre si pelo teste Tukey $(\mathrm{P}>0,05)$.

\section{CONCLUSÃO}

As principais alterações bioquímicas, hematológicas e hemogasométricas verificadas em bezerros infectados experimentalmente com $10^{8}$ UFC de Salmonella Dublin foram reduções das concentrações de albumina, ferro, glicose, magnésio e proteína total, aumento do teor plasmático de fibrinogênio, leucocitose, acidose metabólica e hiponatremia.

\section{AGRADECIMENTOS}

Os autores agradecem à Fundação de Amparo à Pesquisa do Estado de São Paulo (FAPESP), pelo suporte financeiro e pelas bolsas de pesquisa, e à Fundação Oswaldo Cruz, pelo fornecimento da cepa de Salmonella Dublin.

\section{REFERÊNCIAS BIBLIOGRÁFICAS}

BARRINGTON， G.M.; GAY， J.M.; EVERMANN, J.F. Biosecurity for neonatal gastrointestinal diseases. Vet. Clin. N. Am.: Food Anim. Prat., v.18, p.7-34, 2002.

CONSTABLE, P.D.; GOHAR, M.; MORIN, D.E. Use of hypertonic saline dextran soluction to resuscite hypovolemic calves with diarrhea. Am. J. Vet. Res., v.57, p.97-104, 1996.

FEITOSA, F.L.F. Semiologia veterinária: a arte do diagnóstico. 1.ed. São Paulo: Roca, 2004. $807 \mathrm{p}$.

GODSON, D.L.; BACA-ESTRADA, M.E.; VAN KESSEL, A.G. Regulation of bovine acute phase responses by recombinant interleukin-1ß. Can. J. Vet., v.59, p.249-255, 1995. 
KANEKO, J.J.; HARVEY, J.W.; BRUSS, M.L. Clinical biochemistry of domestic animals. 6.ed. San Diego: Academic, 2008. 916p.

MILLAR, H.R.; SIMPSON, J.G.; SRALKEN, A.L. An evaluation of the heat precipitation method for plasma fibrinogen estimation. $J$. Clin. Pathol., v.24, p.827-830, 1971.

RICE, D.H.; BESSER, T.E.; HANCOCK, D.D. Epidemiology and virulence assessment of Salmonella Dublin. Vet. Microbiol., v.56, p.1124, 1997.

SANTOS, R.L.; TSOLIS, R.M.; ZHANG, S. et al. Salmonella-induced cell death not required for enteritis in calves. Infect. Immun., v.69, p.4610-4617, 2001.

SANTOS, R.L.; TSOLIS, R.M.; BAÜMLER, A.J. et al. Hematologic and serum biochemical changes in Salmonella ser Typhimuriuminfected calves. Am. J. Vet. Res., v.63, p.11451150, 2002.

SARWARI, A.R.; MAGDER, L.S.; LEVINE, P. et al. Serotype distribution of Salmonella isolates from food animals after slaughter differs from that isolates found in humans. $J$. Infect. Dis., v.183, p.1295-1299, 2001.

SLANINA, L.; BOMBA, A.; LEHOCKÝ, J. et al. Hemoconcentration in calves and its relation to the hematologic, protein, mineral and electrolyte profile. Vet. Med., v.29, p.425-434, 1984.

SMITH, B.P.; HABASHA, F.; REINAGUERRA, M. et al. Bovine salmonellosis: experimental production and characterization of the disease in calves, using oral chalenge with Salmonella Typhimurium. Am. J. Vet. Res., v.40, p.1510-1513, 1979.
SMITH, B.P.; OLIVER, D.G.; SINGH P. et al. Detection of Salmonella Dublin mammary gland infection in carrier cows, using an enzyme-linked immunosorbent assay for antibody in milk or serum. Am. J. Vet. Res., v.50, p.1352-1360, 1989.

THRALL, M.A. Hematologia e Bioquímica Clínica Veterinária. 1.ed. São Paulo: Roca, 2007. 592p.

VELING, J.; BARKEMA, H.W.; SCHANS, I. et al. Herd-level diagnosis for Salmonella enterica subsp. enterica serovar Dublin infection in bovine dairy herds. Prev. Vet. Med., v.53, p.31$42,2002$.

WALKER, P.G.; CONSTABLE, P.D.; MORIN, D.E. et al. A realible, practical and economical protocol for inducing diarrhea and severe desidratation in the neonatal calf. Can. J. Vet. Res., v.62, p.205-213, 1998.

WRAY, C. Some haematological and blood biochemical findings during experimental Salmonella Typhimurium infection in calves. Zentbl. Veterinarmed B, v.27, p.365-373, 1980.

WRAY, C.; DAVIES, R.H. Salmonella infections in cattle. In: WRAY, C.; WRAY A. (Eds.). Salmonella in domestic animals. Oxon: CABI Publishing, 2000. p.169-191.

ZAR, J. H. Biostatistical analysis. 4.ed. New Jersey: Prentice Hall, 1999. 663p. 